\title{
A peculiar thermonuclear X-ray burst from the transiently accreting neutron star SAX J1810.8-2609
}

\author{
N. Degenaar ${ }^{1} \dagger$ and R. Wijnands ${ }^{2}$ \\ ${ }^{1}$ University of Michigan, Dept. of Astronomy, 500 Church St, Ann Arbor, MI 48109, USA \\ email: degenaar@umich.edu \\ ${ }^{2}$ Astronomical Institute "Anton Pannekoek", University of Amsterdam \\ Postbus 94249, 1090 GE Amsterdam, The Netherlands \\ email: r.a.d.wijnands@uva.nl
}

\begin{abstract}
We report on a thermonuclear (type-I) X-ray burst that was detected from the neutron star low-mass X-ray binary SAX J1810.8-2609 in 2007 with Swift. This event was longer $\left(\simeq 20 \mathrm{~min}\right.$ ) and more energetic (a radiated energy of $E_{b} \simeq 6.5 \times 10^{39} \mathrm{erg}$ ) than other $\mathrm{X}$-ray bursts observed from this source. A possible explanation for the peculiar properties is that the X-ray burst occurred during the early stage of the outburst when the neutron star was relatively cold, which allows for the accumulation of a thicker layer of fuel. We also report on a new accretion outburst of SAX J1810.8-2609 that was observed with MAXI and Swift in 2012 . The outburst had a duration of $\simeq 17$ days and reached a $2-10 \mathrm{keV}$ peak luminosity of $\simeq 3 \times$ $10^{37}(D / 5.7 \mathrm{kpc})^{2} \mathrm{erg} \mathrm{s}^{-1}$. This is a factor $>10$ more luminous than the two previous outbursts observed from the source, and classifies it as a bright rather than a faint X-ray transient.
\end{abstract}

Keywords. stars: neutron, X-rays: binaries, X-rays: bursts, X-rays: individual (SAX J1810.82609)

\section{Introduction}

SAX J1810.8-2609 is a transient neutron star LMXB that was discovered by BeppoSAX on 1998 March 10 when it exhibited an accretion outburst (Ubertini et al. 1998). BeppoSAX and ROSAT observations detected the source at a luminosity of $L_{X} \simeq(0.2-1) \times 10^{36}(D / 5.7 \mathrm{kpc})^{2} \mathrm{erg} \mathrm{s}^{-1}(2-10 \mathrm{keV})$, and suggest that the outburst had a duration of $\gtrsim 13$ days (Greiner et al. 1999; Natalucci et al. 2000).

Soon after its discovery, a type-I X-ray burst was detected from SAX J1810.8-2609 (Cocchi et al. 1999; Natalucci et al. 2000). These thermonuclear explosions occur on the surface of accreting neutron stars due to unstable burning of helium/hydrogen. The majority of observed X-ray bursts have a duration of $\simeq 10-100 \mathrm{~s}$ and generate a radiated energy output of $E_{b} \simeq 10^{39} \mathrm{erg}$ (e.g., Galloway et al. 2008; Chelovekov \& Grebenev 2011). Occasionally intermediately-long X-ray bursts are observed, which are more energetic $\left(E_{b} \simeq 10^{40-41} \mathrm{erg}\right.$ ) and longer (tens of minutes) than normal X-ray bursts (e.g., in 't Zand et al. 2008; Falanga et al. 2008; Linares et al. 2009; Degenaar et al. 2010, 2011).

Renewed activity was detected from SAX J1810.8-2609 with Swift, INTEGRAL and RXTE in 2007 August (Parsons et al. 2007; Degenaar et al. 2007; Haymoz et al. 2007; Fiocchi et al. 2009). During this outburst INTEGRAL detected 17 X-ray bursts, which had an observed duration of $\simeq 10-30 \mathrm{~s}$ (3-25 keV; Fiocchi et al. 2009; Chelovekov \& Grebenev 2011). The brightest event reached a bolometric peak flux of $F_{\text {peak }} \simeq 1 \times$ $10^{-7} \mathrm{erg} \mathrm{cm}^{-2} \mathrm{~s}^{-1}$, implying a distance of $D \leqslant 5.7 \mathrm{kpc}$ (Fiocchi et al. 2009).

$\dagger$ Hubble fellow 
Figure 1. Swift/XRT light curve showing the decay of the X-ray burst detected from SAX J1810.8-2609 on 2007 August 5 (BAT trigger 287042). The black and grey data points indicate $\mathrm{WT}$ and $\mathrm{PC}$ mode data, respectively. The dashed curve shows a fit to a power-law decay with an index of $\alpha=-1.43$, and the solid line a fit to an exponential function with a decay time of $\tau=129 \mathrm{~s}$. The dotted horizontal line indicates the persistent emission level.

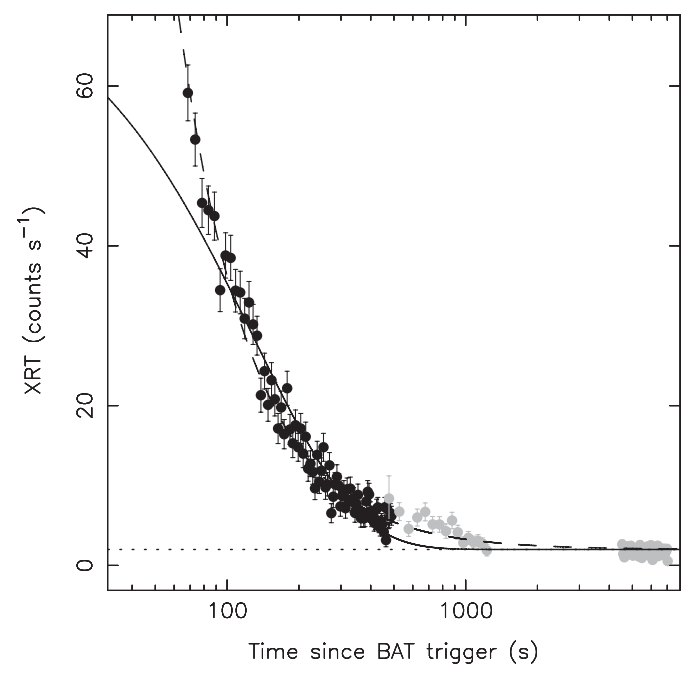

\section{A long thermonuclear X-ray burst detected with Swift in 2007}

On 2007 August 5 at 11:27:26 UT, the Swift/BAT was triggered by SAX J1810.8-2609 (trigger 287042; Parsons et al. 2007). We investigated the trigger data and follow-up XRT observations, and conclude that the BAT triggered on a type-I X-ray burst. For the details on the reduction and analysis procedures we refer to Degenaar et al. (2012a). We carried out exactly the same analysis for SAX J1810.8-2609.

The BAT light curve shows a single $\simeq 10$-s long peak. The average spectrum can be described by a black body model with a temperature of $k T_{b b} \simeq 3.0 \mathrm{keV}$ and an emitting radius of $R_{b b} \simeq 7 \mathrm{~km}$ (Table 1 ), which is typical for the peak emission of X-ray bursts. We estimate a bolometric peak flux of $F_{\text {peak }} \simeq 7 \times 10^{-8} \mathrm{erg} \mathrm{cm}^{-2} \mathrm{~s}^{-1}(0.01-100 \mathrm{keV})$, which is similar to that observed for other X-ray bursts of SAX J1810.8-2609 (Cocchi et al. 1999; Natalucci et al. 2000; Fiocchi et al. 2009; Chelovekov \& Grebenev 2011).

Automated follow-up XRT observations commenced $\simeq 65 \mathrm{~s}$ after the BAT trigger. The XRT light curve shows a continuous decay in count rate until it settles at a constant level $\simeq 1200 \mathrm{~s}$ after the BAT trigger (Figure 1 ). The light curve can be described by an exponential with a decay time of $\tau \simeq 129 \mathrm{~s}$, but a power law with a decay index of $\alpha \simeq-1.43$ provides a better fit (Figure 1). The XRT data can be described by a black body model that cools along the decay (Table 1), a typical signature of X-ray bursts.

The total estimated fluence of the X-ray burst inferred from the BAT and XRT data

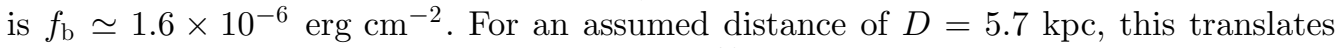
into a total radiated energy of $E_{b} \simeq 6.5 \times 10^{39} \mathrm{erg}$. We use $\simeq 2 \mathrm{ks}$ of XRT PC mode data obtained between $\simeq 4000-6000 \mathrm{~s}$ after the BAT trigger to characterize the persistent accretion emission at the time of the X-ray burst (Figure 1). This data can be described by a simple absorbed power law model with $N_{H}=(6.2 \pm 0.1) \times 10^{21} \mathrm{~cm}^{-2}$ and $\Gamma=2.4 \pm 0.3$. We estimate a bolometric accretion luminosity of $L_{a c c} \simeq 5 \times 10^{35}(D / 5.7 \mathrm{kpc})^{2} \mathrm{erg} \mathrm{s}^{-1}$. The characteristics of the X-ray burst and persistent emission are summarized in Table 1.

SAX J1810.8-2609 was covered by the RXTE/PCA Galactic bulge scan project between 1999 February 5 and 2011 October 30 (Swank \& Markwardt 2001), which reveals one outburst from the source (in 2007). The source was detected above the background level $\left(L_{X} \gtrsim 3 \times 10^{35} \mathrm{erg} \mathrm{s}^{-1}\right)$ between 2007 August 4 and October 28 at an average 2-10 $\mathrm{keV}$ luminosity of $L_{X} \simeq 3 \times 10^{36}(D / 5.7 \mathrm{kpc})^{2} \mathrm{erg} \mathrm{s}^{-1}$. Non-detections on August 1 and November 1 , suggest an outburst duration of $\simeq 85-92$ days. 
Table 1. Time-resolved spectral analysis of the X-ray burst.

\begin{tabular}{lllll}
\hline Instrument & $\Delta t(\mathrm{~s})$ & $k T_{\mathrm{bb}}(\mathrm{keV})$ & $R_{\mathrm{bb}}(\mathrm{km})$ & $F_{\mathrm{bol}}\left(\mathrm{erg} \mathrm{cm} \mathrm{c}^{-2} \mathrm{~s}^{-1}\right)$ \\
\hline BAT & $0-10$ & $3.0 \pm 0.5$ & $7.3_{-2.9}^{+4.9}$ & $4.4 \times 10^{-8}$ \\
$\mathrm{XRT} / \mathrm{WT}$ & $65-100$ & $1.05 \pm 0.04$ & $8.1 \pm 0.2$ & $2.7 \times 10^{-9}$ \\
$\mathrm{XRT} / \mathrm{WT}$ & $101-160$ & $0.90 \pm 0.03$ & $8.0 \pm 0.2$ & $1.4 \times 10^{-9}$ \\
$\mathrm{XRT} / \mathrm{WT}$ & $161-265$ & $0.79 \pm 0.03$ & $7.2 \pm 0.2$ & $6.7 \times 10^{-10}$ \\
$\mathrm{XRT} / \mathrm{WT}$ & $266-485$ & $0.71 \pm 0.03$ & $6.1 \pm 0.2$ & $3.1 \times 10^{-10}$ \\
$\mathrm{XRT} / \mathrm{PC}$ & $492-738$ & $0.69 \pm 0.06$ & $5.1 \pm 0.4$ & $2.1 \times 10^{-10}$ \\
\hline
\end{tabular}

Note. Quoted errors refer to $90 \%$ confidence levels. $\Delta t$ indicates the time since the BAT trigger and $F_{\mathrm{bol}}$ the estimated bolometric flux over the interval. The simultaneous fit resulted in $N_{H}=(2.2 \pm 0.3) \times 10^{21} \mathrm{~cm}^{-2}\left(\chi_{\nu}^{2}=0.93\right.$ for 281 d.o.f. $)$ and we assumed $D=5.7 \mathrm{kpc}$.

\section{A new accretion outburst in 2012 seen with $M A X I$ and Swift}

MAXI monitoring observations show that SAX J1810.8-2609 was again active between 2012 May 7-24. We estimate an average $2-20 \mathrm{keV}$ luminosity of $L_{X} \simeq 6.3 \times$ $10^{36}(D / 5.7 \mathrm{kpc})^{2} \mathrm{erg} \mathrm{s}^{-1}$, peaking at $L_{X} \simeq 2.0 \times 10^{37}(D / 5.7 \mathrm{kpc})^{2} \mathrm{erg} \mathrm{s}^{-1}$. The MAXI data suggests that the source intensity was $L_{X} \gtrsim 10^{36} \mathrm{erg} \mathrm{s}^{-1}$ for $\simeq 17$ days.

A pointed Swift/XRT observation was performed on 2012 May 12 (Obs ID 32459001). The WT spectrum is best described by a combined power law and black body model with $N_{H}=(0.51 \pm 0.02) \times 10^{21} \mathrm{~cm}^{-2}, \Gamma=1.67 \pm 0.05, k T_{b b}=0.74 \pm 0.03 \mathrm{keV}$, and $R_{b b}=13.4 \pm$ $1.4 \mathrm{~km}\left(\chi_{\nu}^{2}=1.09\right.$ for 721 d.o.f. $)$. The resulting unabsorbed $2-10 \mathrm{keV}$ model flux of $F_{X} \simeq$ $6.9 \times 10^{-9} \mathrm{erg} \mathrm{cm}^{-2} \mathrm{~s}^{-1}$ implies a luminosity of $L_{X} \simeq 2.7 \times 10^{37}(D / 5.7 \mathrm{kpc})^{2} \mathrm{erg} \mathrm{s}^{-1}$.

Simultaneously obtained UVOT observations using the $u w 1$ filter $\left(\lambda_{0}=2600 \AA\right)$ reveal an object at R.A. $=18^{\mathrm{h}} 10^{\mathrm{m}} 44.487^{\mathrm{s}}$, decl. $=-26^{\circ} 09^{\prime} 01.30^{\prime \prime}$, with an uncertainty of $0.61^{\prime \prime}$. This coincides exactly with the Chandra position of SAX J1810.8-2609 (Jonker et al. 2004) and suggests that this is the UV counterpart of the LMXB. We determine (Vega) magnitudes of $u w 1=18.80 \pm 0.12$ and $18.57 \pm 0.22$ mag for the two separate exposures.

\section{Discussion}

The X-ray burst from SAX J1810.8-2609 observed with Swift is both longer and more energetic than others observed from the source (Cocchi et al. 1999; Natalucci et al. 2000; Fiocchi et al. 2009; Chelovekov \& Grebenev 2011). The duration is similar to that of intermediately long X-ray bursts, but the radiated energy output is an order of magnitude lower. This suggests that the X-ray burst observed from SAX J1810.8-2609 was a normal $\mathrm{X}$-ray burst, albeit with an unusual long duration. There are different explanations for such peculiar X-ray bursts (see Degenaar et al. 2012a, and references therein).

The long X-ray burst occurred within a few days after the onset of the 2007 accretion outburst. This implies that the neutron star crust had likely not yet been significantly heated due to accretion, and hence the heat flux from the crust towards the surface was small. Combined with a low accretion rate $(\simeq 0.1 \%$ of Eddington), this suggests that the temperature in the accreted envelop was likely low. This allows for the accumulation of a relatively thick layer of fuel before the ignition conditions are met, and may have caused its unusual properties compared to other X-ray bursts observed from the source.

The X-ray flux observed with MAXI and Swift during the 2012 outburst of SAX J1810.8-2609 is a factor of $>10$ higher than seen during its 1998 and 2007 outbursts. Although the previous activity of the source classified it as a faint LMXB (Natalucci et al. 2000; Jonker et al. 2004; Fiocchi et al. 2009), this demonstrates that it is actually a 
Table 2. Characteristics of the X-ray burst and the post-burst persistent emission.

\begin{tabular}{|c|c|}
\hline Parameter & Value \\
\hline $\begin{array}{l}\text { Bolometric accretion luminosity, } L_{\text {acc }} \\
\text { Global mass-accretion rate, } \dot{M}=R_{N S} L_{\text {acc }} / G M_{N S} \\
\text { Local mass-accretion rate, } \dot{m}=\dot{M} / 4 \pi R_{N S}^{2}\end{array}$ & $\begin{array}{l}\simeq 4.9 \times 10^{35} \mathrm{erg} \mathrm{s}^{-1} \\
\simeq 4.1 \times 10^{-11} \mathrm{M}_{\odot} \mathrm{yr}^{-1} \\
\simeq 2.1 \times 10^{2} \mathrm{~g} \mathrm{~cm}^{-2} \mathrm{~s}^{-1}\end{array}$ \\
\hline $\begin{array}{l}\text { Bolometric X-ray burst peak flux, } F_{\text {peak }} \\
\text { Exponential decay time, } \tau \\
\text { Powerlaw decay index, } \alpha \\
\text { Total duration, } t_{\mathrm{b}} \\
\text { Total fluence, } f_{\mathrm{b}} \\
\text { Total radiated energy, } E_{\mathrm{b}}\end{array}$ & $\begin{array}{l}\simeq 7 \times 10^{-8} \mathrm{erg} \mathrm{cm}^{-2} \mathrm{~s}^{-1} \\
\simeq 129 \mathrm{~s} \\
\simeq 1.43 \\
\simeq 1200 \mathrm{~s} \\
\simeq 1.7 \times 10^{-6} \mathrm{erg} \mathrm{cm}^{-2} \\
\simeq 6.5 \times 10^{39} \mathrm{erg}\end{array}$ \\
\hline
\end{tabular}

Note. The quoted peak flux is unabsorbed and for the 0.01-100 keV energy range. The quoted accretion luminosity and mass-accretion rates were inferred from fitting $\simeq 2 \mathrm{ks}$ of post-burst persistent emission. We assumed a distance of $D=5.7 \mathrm{kpc}$.

bright transient (cf. Wijnands et al. 2006). It is not uncommon for bright X-ray transients to exhibit faint outbursts (e.g., Degenaar \& Wijnands 2009; Degenaar et al. 2012b)

\section{Acknowledgements}

ND is supported by NASA through Hubble Postdoctoral Fellowship grant number HSTHF-51287.01-A from the Space Telescope Science Institute. RW is supported by a European Research Council starting grant. This work made use of Swift data supplied by the UK Swift Science Data Centre at the University of Leicester, MAXI data provided by RIKEN, JAXA and the MAXI team, and publicly available $R X T E / P C A$ bulge scan light curves maintained by C. Markwardt.

\section{References}

Chelovekov, I. V. \& Grebenev, S. A. 2011, Astronomy Letters, 37, 597

Cocchi, M., Bazzano, A., Natalucci, L., et al. 1999, Astrophysical Letters and Communications, 38,133

Degenaar, N., Jonker, P. G., Torres, M. A. P., et al. 2010, MNRAS, 404, 1591

Degenaar, N., Klein-Wolt, M., \& Wijnands, R. 2007, The Astronomer's Telegram, 1175

Degenaar, N., Linares, M., Altamirano, D., \& Wijnands, R. 2012a, ApJ, 759, 8

Degenaar, N. \& Wijnands, R. 2009, A\&A, 495, 547

Degenaar, N., Wijnands, R., Cackett, E. M., et al. 2012b, A\&A, 545, A49

Degenaar, N., Wijnands, R., \& Kaur, R. 2011, MNRAS, 414, L104

Falanga, M., Chenevez, J., Cumming, A., et al. 2008, A\&A, 484, 43

Fiocchi, M., Natalucci, L., Chenevez, J., et al. 2009, ApJ, 693, 333

Galloway, D. K., Muno, M. P., Hartman, J. M., et al. 2008, ApJS, 179, 360

Greiner, J., Castro-Tirado, A. J., Boller, T., et al. 1999, MNRAS, 308, L17

Haymoz, P., Eckert, D., Shaw, S., \& Kuulkers, E. 2007, The Astronomer's Telegram, 1185, 1

in 't Zand, J. J. M., Bassa, C. G., Jonker, P. G., et al. 2008, A\&A, 485, 183

Jonker, P. G., Galloway, D. K., McClintock, J. E., et al. 2004, MNRAS, 354, 666

Linares, M., Watts, A. L., Wijnands, R., et al. 2009, MNRAS, 392, L11

Natalucci, L., Bazzano, A., Cocchi, M., et al. 2000, ApJ, 536, 891

Parsons, A. M., Barthelmy, S. D., Gehrels, N., et al. 2007, GRB Coordinates Network, 6706

Swank, J. \& Markwardt, C. 2001, in ASP Conf. Series, Vol. 251, New Century of X-ray Astronomy, ed. H. Inoue \& H. Kunieda, 94

Ubertini, P., in 't Zand, J., Tesseri, A., Ricci, D., \& Piro, L. 1998, IAU Circ., 6838, 1

Wijnands, R., in 't Zand, J. J. M., Rupen, M., et al. 2006, A\&BA, 449, 1117 